\title{
ESPÉCIES CONSIDERADAS PLANTAS DANINHAS EM ÁREAS CULTIVADAS NO ESTADO DE MINAS GERAIS
}

M.B. FERREIRA* \& J.P. del C. LACA-BUENDIA**

* Botânica, Pesquisadora da EPAMIG. 30.000 Belo Horizonte, M.G.

** Eng. ${ }^{\circ}$ Agr. ${ }^{\circ}$, Pesquisador da EPAMIG, 30.000 Belo Horizonte, M.G.

Recebido para publicação em 20.03.78

\section{RESUMO}

No Estado de Minas Gerais já foram feitas várias listagens de espécies de plantas daninhas que ocorrem em áreas restritas e diversificadas, sendo que até o presente momento não foi realiza- do um levantamento das mesmas que ocorram em áreas cultivadas.

Foram identificadas cerca de 295 espécies, pertencentes a 29 famílias, representando 158 gêneros; sendo as famílias mais representantes: Compositae, Gramineae e Leguminosae com 61,43 e 32 espécies, respectivamente. 


\section{SUMMARY}

A survey in the main cultivation areas of the State of Minas Gerais resulted in the determination of 295 weed species, from 158 genera and belonging to 29 families; the families presenting a greater number of species are: Compositae, Gramineae and Leguminosae with 61,43 e 32 species, respectively.

\section{INTRODUÇÃO}

Até o presente momento podem ser encontrados apenas trabalhos regiona is em termos de levantamento de plantas daninhas no Estado. Warning (19); Vianna (15); Kuhlman et al (8); Monteiro Filho (12), Monteiro et al (14); Monteiro (13); Vidal \& Vidal (16, 17, 18); Angely (1); Gentchjnicov et al (7); Laca-Buendia et al (9, 10 e 11); Blanco (2, 3 e 4); Ferreira \& Laca-Buendia (6) e D'assumpção et al (5), tratam das plantas daninhas ocorrentes em áreas restritas e diversificadas do Estado, ou en tão, citam Minas Gerais como local de ocorrência de espécies por eles estudadas em outras áreas do país.

\section{MATERIAIS E MÉTODOS}

Para a organização da listagem anexa utilizamos os resultados ocorren tes dos levantamentos já realizados pelos autores e que se acham inclusos em Laca-Buendia et al (9, 10 e 11), Ferreira \& Laca-Buendia (6) e D'assumpção et al (5), acrescidos de outras, adquiridos através de coletas feitas em culturas várias, situadas nas Fazendas Experimentais pertencentes à EPAMIG (35) que se acham localizadas em diferentes regiões do Estado. Após o que foram identificadas segundo os métodos clássicos de taxonomia e incluidas no Herbário da EPAMIG - Belo Horizonte, M.G.. Foram ainda consultados os Herbários do Jardim Botânico do Rio de Janeiro (RB), da Universidade de Brasilia (UB) e de São Paulo (SP).

\section{RESULTADOS}

Foram identificadas cerca de 295 espécies, pertencentes a 29 famílias, representando 158 gêneros, que se acham consignadas em lista anexa.

As famílias mais representativas foram: Compositae, Gramineae e Leguminosae com 61,43 e 32 espécies, respectivamente. 


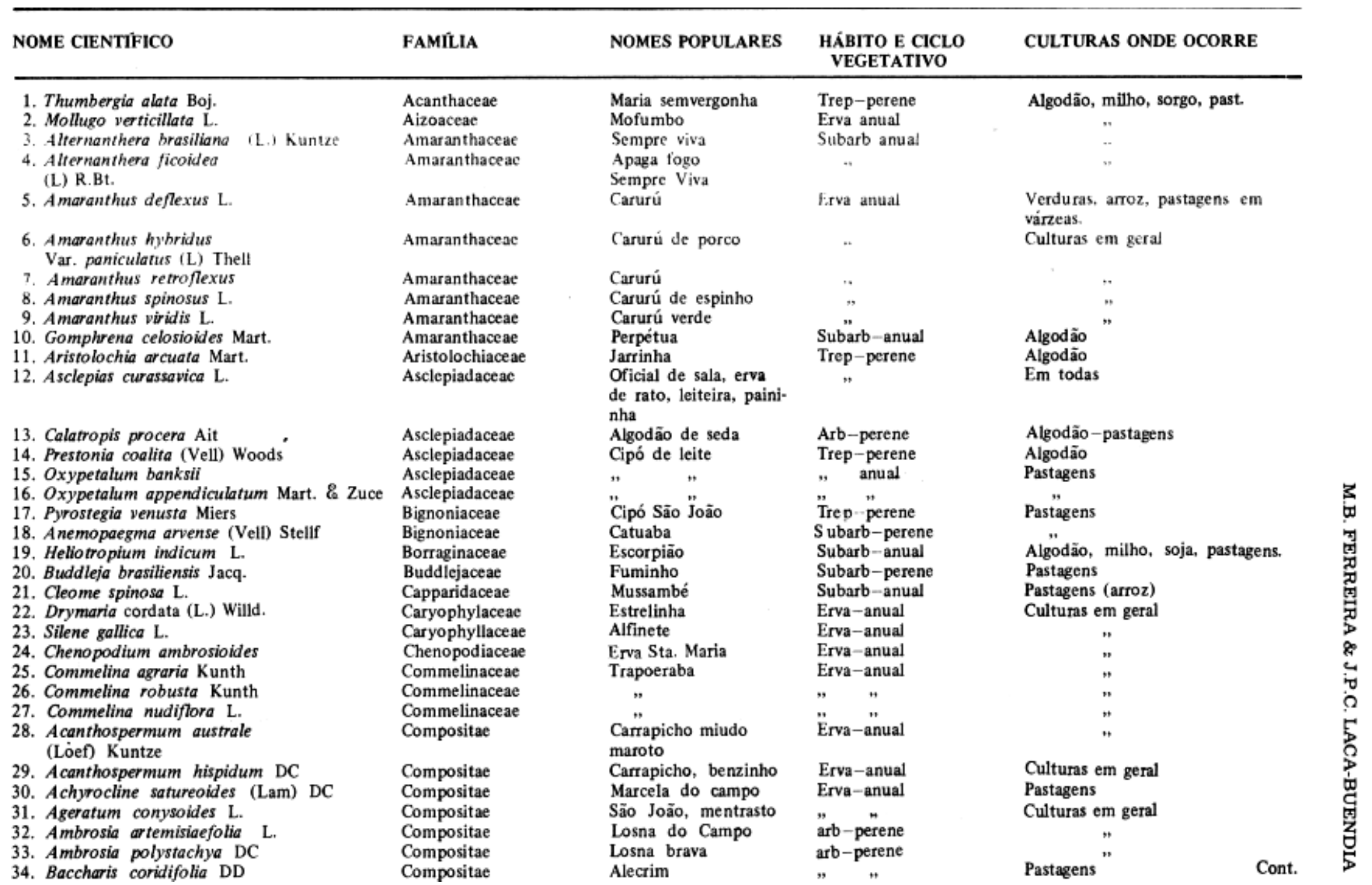


35. Baccharis dracunculifolia

36. Baccharis trimera (Less) DC

37. Baccharis trinervii

39. Bidens segetum Mart ex Colla

40. Blainvillea latifolia

41. Blainvillea biaristata DC

42. Centratherium punctatum Cass.

43. Chaptalia integerrima (Vell) Burk

44. Chaptalia mutans (L.) Polack

45. Cosmus caudatus H.B.K.

46. Eclipta alba (L.) Hassk

47. Elephantopus mollis H. BK

48. Elvira biflora (L.) DC

49. Emilia sagitata (Vahl) DC

50. Emilia sonchifolia DC

51. Erechitites hieracifolia (L.) Rafin

52. Erechitites valerianaefolia DC

53. Erigeron bonariensis L.

54. Eupatorium hirsutum Hook et Arn

55. Eupatorium laevigatum Lam

56. Eupatorium macrocephalium

57. Eupatorium pauciflorum H.B.K

58. Eupatorium purpurascens Schultz - Bip

59. Eupatorium Squalidum DC

60. Galinsoga parviflora Gar

61. Gnaphalium spathulatium Lam.

62. Ganaphalium spicatum Lam

63. Gochnatia polymapha (Less) Cabr

64 Gochnatia velutina (Bong) Cabr

65. Hypocheris brasiliensis Griseb

66. Jaegerio hirta (Lag) Less

67. Mikania cordifolia (L.f) Willd

68. Mikania hirsutissima DC

69. Orthopapus angustifolius (Sw) Gleasson

70. Porophyllum ruderale (Jacq) Cass.

71. Pterocaulon alopecurioides (Lam) DC

72. Schkuria pinata (Lam) O Kuntze

73. Senecio brasiliensis Lam.

74. Siegesbeckia orientalis L.

75. Solidago microglossa DC

76. Sonchus asper (L.) Hill

\section{FAMILIA}

Compositae

Compositac

Compositae

Compositae

Compositae

Compositae

Compositae

Compositae

Compositae

Compositae

Compositae

Compositae

Compositae

Compositae

Compositae

Compositae

Compositac

Compositae

Compositae

Compositae

Compositac

Compositae

Compositac

Compositae

Compositae

Compositae

Compositac

Compositae

Compositae

Compositat

Compositae

Compositae

Compositae

Compositae

Compositae

Compositae

Compositae

Compositae

Compositae

Compositae

Compositae

Composiate

Compositae
NOMES POPULARES HÁBITO E CICLO

VEGETATIVO

Carqueja
Carqueja

Picão

Erva-anual

Roxinha, cravinha "Erva anual

Paraqueda

Paraqueda

Bem-me-quer,

Beijo de moça

Erva-lanceta

Fumo bravo

Erva Estrela

Pincel

serralha

Serralha brava

Serralha brava

Salpeixinho

Cambará

Cambará

Cambará

$"$

Picäo branco

Macela branca

Cambara - açu

Cambará

me leva

E.rva botāo

Cipó cabeludo

Língua de vaca

Cravo do mato

Barbasco

Erva lanceta, flor das Subarb-anual

almas

Botão de ouro

Douradinha

Serralha

Subarb anua

Arb perene

Arb perene

Arb perene

Erva anual

Erva anual

Erva anual

Arb perene

Frva anual

Trep anual

Subarb-perene

Erva anua

Erva anual

Subarb-anual

Erva anual
CULTURAS ONDE OCORRE

Pastagens

Todas

Pastagens

Pastagens, algodão

Algodão, pastagens Pastagens

,

Todas

Algodão, pastagens

Todas

Todas

$"$

Pastagens

Pastagens

Pastagens

Culturas em geral

Pastagens

Pastagens

".

Culturas em geral

Pastagens

$"$

Culturas em geral

Pastagens

Culturas em geral

Cont. 
NOME CIENTIFICO

77. Sonchus oleraceus L

78. Spilanthes acmella L.

79. Synedrella nodiflora Gardn

80. Tagetes minuta L

81. Taraxacum officicinale Weber

82. Vernonia ferruginea Less.

83. Vernonia grandiflora Less.

84. Vernonia polynthes Less.

85. Vernonia remotiflora Ricli.

86. Vernonia scabra Pers

87. Vernonia scorpioides (Lam) Pers.

88. Xanthium cavanillesii Schouw

89. Xanthium spinosum L.

90. Cuscute racemose Wart.

91. Ipomoed acuminata Roen

Compositae

Composiate

Compositae

Compositae

Composiate

Compositae

Composiate

Compositae

Composiate

Compositae

Compositae

Convolvulaceae

Convolvulaceac

92. Ipomoea aristolochiaefolia (H.B.K.) Don. Convolvulacese

93. Ipomoea cynanchifolia Meissn.

94. Ipomoea calrica (L.) Sweet.

95. Ipomoea grandiflora O'Donnel

96. Ipomoea purpurea Lam

97. Merremia aegyptia (L.) Urban

98. Merremia cissoides (Lam.) Hall

99. Merremia dissecta (Jacq Hallier)

100. Merremia macrocalyx (Ruiz et Pav) O'Donnel

101. Quamoclit coccinea Moench

102. Quamoclit pinnata Boj.

103. Brasica campestris L.

104. Lepidium vïginicum L.

105. Cucunis anguria L

106. Luffa operculata L.

107. Momordica charantia L.

108. Carex cryptolepis Maik

109. Cyperus rotundus L.

110. Cyperus distans L. F.

111. Cyperus diffusus Vahl

112. Cyperus esculentus L.

113. Cyperus ferax L.C. Rich

114. Cyperus haspan L.

115. Cyperus imbricatus Retz

116. Cyperus huzulae (L.) Retz

117. Cyperus prollixus Retz

118. Cyperus rivularis Kunth
Convolvulaceac

Convolvulaceae

Convolvulaceae

Convolvulaceae

Convolvulaceae

Convolvulaceac

Convolvulaceae

Convolvulaceae

Convolvilaceae

Convolvulaceae

Cruciferas

Cruciferas

Cucurbitaceae

Cucurbitaceac

Cucurbitaceae

Cyperaceae

Cyperaceae

Cyperaceae

Cyperaceae

Cyperaceac

Cyperaceae

Cyperaceae

Cyperaceac

Cyperaceae

Cyperaceae

Cyperaceae

\section{NOMES POPULARES}

Serralha brava

Agrião Bravo

Picāo brance

Rojẫo-rabo de foguete

Dente de leão

Assa-peixe

Roxinhe

Figo bravo, fumo bravo

Cipó chumbo

Getirana, corda de

viola, amarra amarra

Getirana, corda de

viola, amarra amarra

,

$"$

Getirans

Cipó coração

Cipó esqueleto

mostarda brava

mentrusto

maxixi

bux

Melão de S. Caetano

Tiririca

Tiririca

"

,

$n$

"

$"$

,
HÁBITO E CICLO

VEGETATIVO

Erva anual

Trep anua

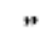

"

$"$

"

"

Trep anus

Trep

Erva anual

Trep anual

$n$

"

Erva perene

"

",

",

$"$

$"$

CULTURAS ONDE OCORRE

Culturas em geral

Pastagens

"

$"$

Culturas em geral

$"$

"

$"$

"

Culturas em geral

"

Pastagens

Culturas em gera

$"$

"

"

"

,

"

, 
119. Dichromena ciliata Vahl

120. Eleocharis nodulosa (Roth) Schultes

121. Fimbristylis complanata Link

122. Fimbristylis dichotona (L.) Vahl

123. Scleria leptoschya Kunth

124. Scleria secans (L.) Urban

125. Euphorbia hypericifolia

126. Euphorbia pilulifera L.

127. Euphorbia prostata Ait

129. Phyllanthus corcovadensis Muell Arg

129. Phyllanthus corcovad

130. Phyllantus niruri L.
131. Ricinus communis L.

132. Andropogon bicornis L.

133. Aristida pallens Cav.

134. Aristida setifolia H.B.K

135. Aristida recurvata H.B.K

136. Aristida adscencionis L.

137. Axonopus barbatus Chase

138. Brachiaria purpurascens Henr.

139. Brachiaria plantaginea (Link) Hich

140. Cenchrus ecninatus L.

141. Cymodon dactylon (L.) Pers

Cyperaceae

143. Digitaria horizontalis Willd.

144. Digitaria sanguinalis (L.) Scop.

145. Echinochloa crusgalli (L.) Beauy.

146. Echinolaena inflexa (Poir) Chas

147. Eleusine indica (L.) Gaertn

148. Eragrostis acuminata Doell

149. Eragrostis ciliaris (L.) R.Br.

150. Eragrostis solida (Ness) Stapf

151. Eragrostis pilosa (L.) Reauv

152. Hyparrhemia rufa (Nees) Stapf

153. Imperata brasiliensis Trin.

154. Melinis minutiflora Reauv.

155. Panicum campestre Nees.

156. Panicum maximum Jacq

157. Paspalum acuminata Raddi

158. Paspalum conjugatum Berg

159. Paspalum conspersum Schrad
Cyperaceae

Cyperaceae

Cyperaceae

Cyperaceac

Cyperaceae

Euphorbiaceae

Euphorbiaceae

Euphorbiaceae

Euphorbiaceae

Euphorbiaceae

Euphorbiaceae

Euphorbiaceae

Gramineae

Gramineae

Gramineae

Gramineac

Gramineae

Gramineae

Gramineac

Gramineae

Gramineae

Gramineae

Gramineae

Gramineae

Capim

Capim navalha

$"$

Erva andorinha

leiteira

Beldroega

Amendoim brave

Quebra pedra

Mamona

Capim de burro

Capim fino

Capim barba de bode

Capim fino

Capim fino

Capim bengo
Capim, papuã, grama

paulist

Benzinho, carrap., timbet

Grama

Pe'de galo, pé de pa-

pagaio

Capim colchão

Gramineae

Gramineae

Gramineae

Gramineae

Gramineae

Gramineae

Gramineae

Gramineac

Gramineac

Gramineae

Gramineae

Gramineae

Gramineae

Gramineae

Gramineae

Gramineae

Gramineae

Gramineae

Gramineae

Capim, arroz, ja

Barbadinho, capim, arro

Capim - flexa

Capim fino

Capim fino

Capim fino

Capim fino

Jaraguá, provisór

Sapé, navalhas

Capim mimoso

Coloniẫo

Grama

Grama - doce

\section{VEGETATIVO}

Culturas em geral

"

$"$

"

$"$

Culturas em geral

Erva "

Erva perene$$
\text { " }
$$$$
"
$$

Erva anual

Erva anual

Erva anual

Capim milhā, capim Erva perene

Pastagens

Pasto em várzeas, arroz

Culturas em geral

Pastagens, algodão

Culturas em geral

Pastagens

Culturas em geral

Culturas em geral

,

Pastagens

Culturas em geral

Pastagens

Culturas em geral

Amendoim, alfafa, arroz, café

milho, algodão, soja, pomares

Arroz, pastagens em várzeas

Arroz, pastagens em geral

Cont. 
FAMILIA

Gramineae

Gramineae

Gramineae

Gramineae

Gramineae

Gramineae

Gramineac

63. Paspalum paniculatum L.

165. Paspalum urvillei Steud

Pennisetum clandestinum Hochst
S. Chiov.

167. Pennisetum setosum (Swartz) L.

168. Rhychelitr
et Hubb.

169. Setaria geniculada (Lam) Beauv.

170. Setaria verticillata (L.) Beauv.

171. Shorghum halepense (L.) Pers.

172. Sporobulus indicus (L.) R.Br.

173. Trichachne insularis (L.) Nees

174. Tristachya Chysolvix Nees. Gramineae

175. Hyptis lophanta Mart. ex. Benth Labiatac

Gramineae

Gramineae

Gramineae

Gramineae

Gramineae

Gramineae

177. Hyptis nudicaulis Benth

Labiatae

Labiatae

178. Leonotis nepetaefolia $\mathrm{R}$. Br.

Labiatae

179. Leonurus sibiricus L.

180. Marsypianthes chamaedrys (Vahl)

181. Acacia plumosa Lowe Kuntze

182. Aeschynomene Falcata DC

183. Aeschynomene selloi Vog

184. Cassia alata L.

185. Cassia basifolia Vog

186. Cassia chartacea Irwim

187. Cassia Colutoides L.

188. Cassia flexuosa $\mathrm{L}$.

189. Cassia patellaria DC

190. Cassia occidentalis L

191. Cassia riparia H.B.K.

192. Cassia rotundifolia Pers

193. Cassia tora $\mathbf{L}$.

194. Crotalaria anagyrioides H.H.K.

195. Crotalaria incana $\mathrm{L}$

196. Crotalaria mucronata Desv

197. Crotalaria stipularia Desv

198. Crotalaria mucronata Desv.

199. Desmodium adscendens (Sw) DC

200. Desmodium barbatum (L.) Benth

Labiatae

Labiatae

\section{NOMES POPULARES}

HÁBITO E CICLO VEGETATIVO

Leguminosae

Leguminosae

Leguminosae

Leguminosae

Leguminosae

Leguminosae

Leguminosae

Leguminosae

Leguminosae

Leguminosae

Leguminosae

Leguminosae

Leguminosae

Leguminosae

Leguminosae

Leguminosae

Leguminosae

Leguminosae

Leguminosae

Leguminosae
Capim de roça

Milhä roxo

Grama batatais

Capim de birro

Cuiabano

Capim arroz

Kikuyo

Rabo de raposa
Capim favorito,

Capim natal

Capim rabo de rato

Capim rabo de raposa

Capim maçaranduv

Capim capeta

Capim amargoso

Capim aveia

Hortelä

Vareta

Hortel

Cordas de São Francis- Subarb anual

"

”

$"$

$"$

Erva perene

Erva anual

Erva anual

Erva anual

Erva perene

Erva anual

Erva perene

Subarb" perene

$$
\text { " }
$$

Cordas de feira

Vassoura

Mimosa, arranha gato

Carrapicho

Carrapicho

Fedegoso do grande

Canudo do pito Fede-

Canso

Peninha

Fedegoso

coração

Mata pasto

guseiro

Guiseiro

,"

Carrapicho
Subarb anual

Arb perene

Subarb perene

Arb perene

Subarb perene

Arbusto perene escan-

dente

Subarb perene

Subarb perene

Subarb perene

Subarb anual

Subarb anual

$"$

Subarb perene
CULTURAS ONDE OCORRE

Culturas em geral

,

Pastagens em várzeas, arroz

Culturas em geral

Pastagens

Culturas em geral

Pastagens

Pastagens

Pastagens

Pastagens, algodão

Pastagens

Culturas em geral

Pastagens

"

,

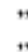

,

$"$

Culturas em geral

Pastagens

Pastagens

Pastagens

"

Culturas em geral

Cont. 
NOMES POPULARES HẢBITO E CICLO

201. Desmodium canum (Gmell) et Thell

202. Desmodium purpureum (Mill) F. et Dner

203. Indigofera hirsuta L.

204. Indigofera lespedizioides H.B.K.

205. Indigofera suffruticosa Mill

206. Mimosa invisa Mart.

207. Mimosa pudica $\mathbf{L}$.

208. Mimosa rixosa Mart.

209. Mimosa velloziana Mart

210. Phaseolus bracteolatus Nees et Mart.

211. Rhynchosia minina DC

212. Zornia dyphylla (L.) Pers

213. Smilax cissoides Mart.

214. Smilax campestris Gris

215. Cuphea balsamona Chamb. S. Schl.

216. Cuphea mesostemon Kohne

217. Bastardia bivalvis $\mathrm{K}$.

218. Bastardia elegans K.

219. Gaya aurea St. Hit.

220. Gaya gracilipes K, Schum

221. Gaya gucrkeana K. Schum

222. Gaya pilosa K. Schum

223. Malvastrum coromandelianum (L.) Garcke

224. Pavonia cancellata Cav.

225. Sida acrantha Link

226. Sida acuta Burm

227. Sida aurantiaca St. Hill Less et Camb.

228. Sida carpinifolia L. F. form carpinifolia H. Mont.

229. Sida cerradoensis Krapov.

230. Sida cordifolia $\mathbf{L}$.

231. Sida glaziovii K. Schum

232. Sida linifolia Cav.

233. Sida martiana S.H.L.

234. Sida micrantha St. Hill

235. Sida rhombifolia L. Var. rhombifolia

236. Sida santaremmensis H. Monteiro

237. Sida spinosa L.

238. Sida tuberculata R.E. Fries Var. pseuso rohombifolia $\mathrm{H}$. Monteiro

239. Sida urens L.

240. Sida viarum St. Hill
Leguminosac

Leguminosac

Leguminosae

Leguminosac

Leguminosae

Leguminosac

Leguminosae

Leguminosae

Leguminosac

Leguminosae

Leguminosae

Leguminosae

Liliaceac

Liliaceae

ythraceae

Lythraceae

Malvaceae

Malvaceae

Malvaceae

Malvaceae

Malvaceae

Malvaceae

Malvaceac

Malvaceac

Malvaceae

Malvaceac

Malvaceae

Malvaceac

Malvaceae

Malvaceae

Malvaceac

Malvaceae

Malvaceae

Malvaceae

Malvaceae

Malvaceae

Malvaceae

Malvaceae

Malvaceae

Malvaceae

Malvaceae

\section{Carrapicho beiço de boi}

Anileira

Analeira

Analeira

Arranha gato

Arranha gato

Feijão bravo

Feijãozinho

Chapinha

alsaparilha

Pć de pinto

Malvão

Malva

"

$"$

Guanxuma, vassoura

Papoula do campo

Malva

Malva

Relógio, malva

Malva baixa

Malva vermelha

Malva branca

Malva guaxima

Malva fina

Guaxima

Malvona

Relógio, guaxima, vas-

soura

Guanxum

Malva

vassoura, malva

Arb perene

"

"

Subarb" perene

Trep anual

Trep anual

Sub anual

Arb escand. perene

Subarb" anual

Subarb" anual

Subarb anual

",

Arb anual

Subarb perene

Subarb anual

Subarb perene

Subarb perene

vassoura

vassoura

Subarb" perene

"

Subarb anual

Subarb" perene
Malicia, dormidcira
VEGETATIVO

CULTURAS ONDE OCORRE

焉
Pastagens

Pastagens, algodão

"

Culturas em geral

,

"

,

Pastagens

Todas as culturas

Pastagens

Pastagẹns

$"$

turas em geral

Curas em geral

$"$

Pastagens

Cont. 
241. Sida lohata L.

242. Wissadula amplissima (L.) R. E. Fries

243. Wissadula subpeltata (Kuntze) R.E. Fries

244. Cissampelos ovalifolia DC

245. Cissampelos glaberrima St. Hill

246. Mirabilis jalapa L

247. Boerhavia decumbens Bahl

248. Oxalis corniculata L

249. Oxalis oxyptera Prog

250. Oxalis refracta $\mathrm{St}$.

252. Polygala paniculata L.

253. Polygala violace

254. Polygonum acre $\mathrm{H}$. B. KZ

255. Polvgonum punctatum Eil

256. Portulacca oleracea $\mathrm{L}$.

257. Talinum patens (Jacq) Willd

258. Borreria alata (Aubl)

259. Borreria capitata (R et P.) DC

260. Borrerio suaveolens G.F.W. Mey

261. Borreria valerianoides Cham et Sch

262. Borreria verbenoides Cham et Schl

263. Borreria verticillata (L.) G.F.W.Mey

264. Manettia cordifolia L.

265. Palicourea marigravii St. Hi

266. Richardia brasiliensis Gome

267. Cardiospermum halicacabum L.

268. Serjanea ereta Radlk

269. Scoparia dulcis L.

270. Cestrum axillare Vel

271. Datura stramonium Lam

272. Nicandra physaloides

273. Physalis angulata $\mathrm{L}$.

274. Solanum aculeatissimum Jacq

275. Solanum americanum L

276. Solanum capsicostrum Link.

277. Solanum erianthum D. Don

278. Solanum lycocarpum St. Hil

279. Solanum palinacanthum

280. Solanum Sisymbrüfolium Lam.

281. Walteria communis St. Hil

282. Walteria indica $\mathrm{L}$.

283. Melochia hermanioides St. Hil

\section{Malvaceae}

Malvaceac

Menispermanae

Manispermanae

Nyctagenaceac

Nyctagenaceac

Oxalidaceae

Oxalidaceae

Oxalidaceac

Plantaginaceae

Polygalaceae

Pclygalaccae

Polygonaceae

Polygonaceae

Portulacaceae

Portulacace:

Rubiaceae

Rubiaceae

Rubiaceac

Rubiaceae

Rubiaceae

Rubiaceae

Rubaiceae

Rubiaceae

Sapindaceae

Sapindaceae

Scrophulariaceae

Solanaceae

olanaceae

Solanaceae

Solanaceae

Solanaceae

Solanaceae

Solanaceae

Solanaceae

Solanaceae

Solanaceae

Solanaceac

Sterculiaceae

Sterculiaceae

Sterculiaceae
Malva rosa

Malva do bico

Malva do bico

Abutua

Cipó de cobra

Maravilha

Beldroega de grand

Trevo

Trevo

Trevo

Tanchagem

Sete - sangria

violeta

Erva do bicho

Beld̈roege

Piolho

Hortelã

$"$

Hortelã

Hortelã

Campanha

Erva café

Estralados

Balão

Tinguí

Vassoura

Coerana café bravo

Erva do diabo

Balāo

Balão rajado

Joá tajado

Maria preta

Joá vermelho

Joá bravo

Fruta de lobo

Joá Bravo

Joá de capote

Malvão

Malvão

Malva
Subarb perene$$
\text { " }
$$

Subarb perene

Trep perene

Arb perene

Subarb anual

Erva anual

"

Subarb perene

Subarb anual

Subarb perene

Erva anual

Subarb" perene

$$
\text { " }
$$

Subarb" perene

Subarb perene

Trep anual

Arb perene

Erva anual

Trep. anual

Arbusto escandente

perene

Subarb anual

Arb perene

Subarb anual

"

"

,

Arb perene

$$
\text { pere }
$$

b anua

Subarb perene

Subarb" anual
Todas as culturas

Pastagens, algodão

Pastagens, algodão

Pastagens

Pastagens, algodão

Pastagens

Culturas em geral

$$
" \text {. }
$$

,

Pastagens

Pastagens

Arroz, pastagens em várzeas

Culturas em geral

Pastagens

",

",

Pastagens

Culturas em geral

Pastagens

Culturas em geral

Pastagens

Culturas em geral

Pastagens

",

"

"

"

Culturas em geral

Pastagens

Cont.

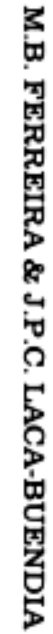




\section{NOME CIENTÍFICO}

284. Melochia pyramidata $\mathrm{L}$

285. Corchorus hirtus L.

286. Trumphetta Bartramia L.

287. Triumphetta semitriloba Jace

288. Hydrocotyle umbellata $\mathrm{L}$.

289. A pium leptophyllum (Pres) Mull

290. Lantana camara L.

91. Lantana lilacina

291. Lantana Macina Desf.

292. Lantana nivea Vent.
293. Stachytarphetta Cayenensis (L.C. Rich)

293. Stachytd

$$
\text { Vahl }
$$

294. Verbena montevidensis Spr.

295. Cissus scabra Baker

\section{FAMILIA}

Sterculiaceae

Tiliaceae

Tiliaceae

Umbelliferae

Verbenaceae

Verbenaceac

Verbenaceae

Verbenaceac

Verbenaceac

Vitaceae

\section{NOMES POPULARES}

HÁBITO E CICLO

VEGETATIVO

Vassoura

Carrapicho

Para - so

Gertrudes

Camará de espinho

Camará rosa

Camará branco

Gervâo

Hortelâ

Uva brava
CULTURAS ONDE OCORRE

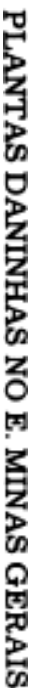




\section{LITERATURA CITADA}

1. Angely, J. Flora analitica e ntogeneografica do Estado de Sáo Paulo, S.P. Ediçōes Phyton, 6 vol., 1. a edição, 1969.

2. Blanco, H.G. Catálogo das espécies de mato infestantes de áreas cultivadas no Brasil Gramíneas perenes. O Biológico, 41: 130143, 1975.

3. Blanco, H.G. Catálogo das espécies de mato infestantes de áreas cultivadas no Brasil - Familia do picáo-preto (Compositae). O Biológico. 42: 62-97, 1976.

4. Blanco, H.G. Catálogo das espécies de mato infestantes de áreas cultivadas no Brasil - Familia das "Guanxumas" (Malvaceae). O Biológico, 42 (9-10): 185-200, 1976.

5. D'assumpçāo, W.R.C.; Ferreira, M.B.; LacaBuendia, J.P. del C. \& Saturnino, H.M. Levantamento das plantas invasoras de pastagens no Estado de Minas Gerais e recomendaçōes para sua erradicaçáo. EPAMIG, Belo Horizonte, M.G., 1973. 63p.

6. Ferreira, M.B. \& Laca-Buendia, J.P. del C. Levantamento $\mathrm{e}$ análise quantitativa das plantas invasoras ocorrentes em culturas na área da fazenda experimental de Santa Rita - EPAMIG, MG. In: Congresso Nacional de Botânica, 28, Belo Horizonte, M.G., 1977. Resumos, p.15.

7. Gemtchujnicov, I.; Almeida, F.M. de \& Gomes, P.F. Levantamento fitossociológico de populaçóes de plantas daninhas em cafezais atacadas por Hemileia vastatrix. Anais do XXIII Congresso Nacional da Sociedade Botânica do Brasil, 205-223, 1972.

8. Kuhl Mann, J.G., Occhioni, P. \& Falcão, J.I. de A. Contribuição ao estudo das plantas ruderais do Brasil. Arquivo do Jardim Botânico do Rio de Janeiro, 7: 43-133, 1947.

9. Laca-Buendia, J.P. del C., Ferreira, M.B. \& Gavilanes, M.L. Contribuiçáo para o conhecimento das ervas daninhas nas principais re-
M.B. FERREIRA \& J.P.C. LACA-BUENDIA

giōes algodoeiras do Estado de Minas Gerais - I. Triângulo Mineiro. In: Projeto Algodáo - Relatório Anual 73/74, EPAMIG. Belo Horizonte, M.G., 63-75 p., 1975a.

10. Laca-Buendia, J.P. del C., Ferreira, M.B. \& Gavilanes, M.L. Contribuição para o conhecimento das ervas daninhas nas principais regióes algodoeiras em áreas de cerrado. Cerrado. 7 (8) : 28-33, 1975b.

11. Laca-Buendia, J.P. del C., Ferreira, M.B. \& Gavilanes, M.L. Levantamento das ervas daninhas nas principais regióes algodoeiras no Estado de Minas Gerais: In: Projeto Algodão - Relatório anual 74/75, EPAMIG, Belo Horizonte, M.G. 103-120 p., 1977.

12. Monteiro Filho, H. da C. Malvaceae Fluminensis invasoras de culturas. In: Seminário Brasileiro de Herbicidas e Ervas Daninhas, 1.', Rio de Janeiro, R.J., 1956. Resumos, p. 125-194.

13. Monteiro, H. da C. Malvaceae Mineiras invasoras de culturas. In: Seminário Brasileiro de Herbicidas e Ervas Daninhas, $2 .^{\circ}$, Rio de Janeiro, R.J., 1958. Resumos, p. 125-131.

14. Monteiro, H.F.; Paixão, J.C. \& Monteiro, J.M. Plantas herbáceas invasoras de cultivos. In: Anais do Seminário Brasileiro de Herbicidas e Ervas Daninhas, $1^{\circ}$, Rio de Janeiro, R.J., 1956. Resumos, p. 157-169.

15. Vianna, U. de S. Montes Claros. Breves apontamentos históricos, geographicos e descriptivos. Belo Horizonte, M.G., 1916. 349p.

16. Vidal, W.R. \& Vidal, M.R.R. Catálogo ilustrado da flórula de Viçosa. Experimentae, 6 (5): 145-225, 1966.

17. Vidal, M.R.R. \& Vidal, W.R. Florula de Viçosa. I - Chenopodiaceae e Amaranthaceae. Revista Ceres, 14 (78): 46-79, 1967.

18. Vidal, M.R.R. \& Vidal, W.R. Plantas ruderais de Viçosa. II - Graminae. Experimentae, 12 (2): 33-61, 1971.

19. Warning, E. Lagoa Santa. Traduçáo de Al bert Leěfgren. Imprensa Oficial. Belo Hori zonte, M.G., 1908. 